\title{
Research on the Algorithm of Simulation Location and Mapping of
}

\section{Mobile Robot}

\author{
Qunying Chen
}

${ }^{1}$ Xi'an Peihua University, Xi'an, 710125, China

Keywords:Mobile robot, Simulation location and mapping, autonomous navigation

\begin{abstract}
The simultaneous localization and mapping (SLAM) of mobile robot is the basic problem and hot spot in the field of robotics, and is also the key to realize autonomous navigation and control decision. This paper first introduces the source of the SLAM problem, and gives different solutions of the problem, including Kalman Filter method, Extended Kalman Filter method and Particle Filter method. The advantages and disadvantages of the three methods are discussed in the paper. Finally, this paper points out the research directions of SLAM problem to provide some reference for the relative researchers.
\end{abstract}

\section{Introduction}

In recent years, more and more attention has been paid to the research of robot. With the development of computer technology and artificial intelligence, intelligent autonomous mobile robot has become an important research direction and research focus in the field of robotics. Localization and mapping of mobile robot is a hot research topic in the field of autonomous mobile robot. There are a number of practical solutions to the robot's self-localization and map location in known environments. However, in many environments, the robot cannot use the global positioning system to locate, and it is very difficult or impossible to obtain the map of the robot working environment in advance. At this time, the robot needs to create a map in a completely unknown environment under the condition of uncertain position. This is the problem of simultaneous localization and mapping of mobile robots.

The mobile robot map building is to build the space model of the surrounding environment of the robot, and to guide the robot navigation or positioning. In order to get the map, the robot must have an external sensor to perceive the external environment. However, these sensors have measurement errors, and the general field of vision is limited, so that the robot must move while building the map. The motion control of the robot provides a wealth of information for the construction of the map, because they contain the location information of the robot during each observation. The robot motion has the same error, only the motion control information is not enough to determine the pose of the robot. Therefore, the map building process depends on the precise position and orientation information, and the robot localization process depends on the accurate map information, the two are both contradictory and relevant, and must be considered simultaneously. The SLAM problem is studied in order to solve the problem of robot localization based on airborne sensors in the absence of precise global positioning sensors. Such a positioning system can meet the requirements of mobile robot navigation in unknown environments. 


\section{Solutionsof the Problem of SLAM}

Kalman Filter Method. KalmanFiltering is an optimal estimation algorithm for the state of the system by using the linear system state equation and the input and output data of the system. Due to the influence of noise and interference in the observation data, the optimal estimation can also be regarded as the filtering process. Kalman, who was visiting the Ames Research Center, found that his method was useful for solving the orbital prediction of the Appollo plan, which was later used in the navigation of the spacecraft. Data filtering is to remove noise and a data processing technique of real data reduction, Kalman filter can from a series of measurement noise variance in measuring the condition of known data, state estimation of dynamic systems. Because it is convenient for computer programming, and can be collected on the spot data update and real-time processing, Kalman filtering is the most widely used in filtering method, communication, navigation, guidance and control in many fields has been well used. Autonomous mobile robot in simultaneous localization and map building when the 6 relates to the processing of environmental information and the uncertainty of sensor noise is unknown and the general use of the probability theory of Kalman Filter to describe the problem. It is an optimal linear recursive estimation algorithm. It cannot completely contain from a series of noise. The basic KF must be applied in accordance with the Gauss distribution of discrete time linear dynamic system, however, in many practical situations, the system state equation and the observation equation is nonlinear, which need to be improved to adapt to the dynamic system of KF nonlinear.

Firstly, we choose the following equations to express the transformation equation and the observation equation.

$$
\begin{aligned}
& \mathrm{x}_{\mathrm{k}}=\mathrm{F}_{\mathrm{k}} \mathrm{x}_{\mathrm{k}-1}+\mathrm{B}_{\mathrm{k}} \mathrm{u}_{\mathrm{k}}+\mathrm{w}_{\mathrm{k}} \\
& \mathrm{z}_{\mathrm{k}}=\mathrm{H}_{\mathrm{k}} \mathrm{x}_{\mathrm{k}}+\mathrm{w}_{\mathrm{k}}
\end{aligned}
$$

In the above formulations, we have:

$$
x_{k} \in R^{n}, z_{k} \in R^{m}, w_{k} \in R^{n}, v_{k} \in R^{m}, F_{k} \in R^{n \times n}, F_{k} \in R^{n \times n} . u_{k} \in R^{l} \text { and } H_{k} \in R^{m \times n} \text {. }
$$

Extended Kalman Filter Method.The Extended Kalman Filter (EKF) is a common nonlinear filtering method, it is the use of Taylor series linearization technique is the most commonly used, the nonlinear filtering problem is transformed into a basic KF approximate linear filtering problem, and then use the linear filter theory, nonlinear filtering problem suboptimal filtering algorithm. EKF is composed of two steps: prediction and update. It is a classical filtering method and a common filtering algorithm in SLAM. As the filtering process inevitably introduces the linearization error, only when the state of the system and the observation model are close to linear, the filtering results can close to the true value, otherwise the linearization error will affect the filtering accuracy is serious, resulting in the divergence. The mapping error occurred.The Extended KalmanFilter is usually used when the state equation or the measurement equation is nonlinear. Kalman initially proposed filtering theory is only suitable for linear systems, Bucy, Sunahara et al. Proposed and studied the Extended Kalman Filter, referred to as EKF.Kalman will be further applied to the field of nonlinear filtering theory. The basic idea of EKF is to linearize the nonlinear system, and then to carry out the Kalman filtering, so EKF is a suboptimal filter. Subsequently, the application and improvement of the two order generalized Kalman filtering method can improve the estimation performance of the nonlinear system by using the filter. Two order filtering method considering Taylor series two times, therefore 
reduce the estimation error caused by the linearization, but greatly increased the amount of computation, so in practice instead of first-order EKF applications.EKF on the nonlinear function of the Taylor expansion to the first order linearization truncation, ignoring the rest of higher order, thus the nonlinear problem into a linear, linear filtering algorithm can be applied to Kalman in nonlinear systems. In this way, the nonlinear problem is solved. EKF has been recognized and widely used in the nonlinear state estimation system. The SLAM problem is usually solved by recursive Bayesian estimation. The formulations of Bayesian estimation and updating are shown as follows:

$$
\begin{gathered}
P\left(x_{k}, m \mid z_{k-1}, u_{k}, x_{0}\right)=\int P\left(x_{k} \mid x_{k-1}, u_{k}\right) P\left(x_{k-1}, m \mid z_{k-1}, u_{k-1}, x_{0}\right) d x_{k-1} \\
P\left(x_{k}, m \mid z_{k}, u_{k}, x_{0}\right)=\frac{P\left(z_{k} \mid x_{k}, m\right) P\left(x_{k}, m \mid z_{k-1}, u_{k}, x_{0}\right)}{P\left(z_{k} \mid z_{k-1}, u_{k}\right)}
\end{gathered}
$$

The two above formulations have given the recursive algorithmof $P\left(x_{k}, m \mid z_{k}, u_{k}, x_{0}\right)$.

Particle Kalman Filter Method.Based on the idea of Monte Carlo method, Particle Filteris the use of particle sets to represent the probability that can be used in any form of state space model. The core idea is to express the distribution of the random state particles from the posterior probability, which is a kind of sequential importance sampling method. In simple terms, the particle filtering method is by looking for a random sample spread in state space to approximate the probability density function, integral operation by sample mean value, and gains the state minimum variance distribution. Here refers to the sample particles, when the sample approximation of the probability density distribution in any form. Although it approximation of the real probability distribution algorithm in distribution, but due to the characteristics of non-parametric, it gets rid of the solution of nonlinear filtering problem of random variables must satisfy the Gauss distribution which can express distribution models more extensively than Gauss, also nonlinear characteristics of variable parameters have the ability of modeling. Therefore, the particle filter can accurately express the posterior probability distribution based on the measurement and control, which can be used to solve the problem. Therefore, particle filter can be applied to any state space model, which is not affected by non-Gauss and nonlinear model. In this paper, the particle filter algorithm is used to solve the nonlinear filtering problem which must be satisfied with the Gauss distribution. The algorithm has been successfully applied to the SLAM problem in recent years.Although only an approximation of the real probability distribution algorithm in distribution, but due to the characteristics of non-parametric, it gets rid of the solution of nonlinear filtering problem of random variables must satisfy the Gauss distribution which can express distribution models more extensively than Gauss, also nonlinear characteristics of variable parameters have the ability of modeling. Therefore, the particle filter can accurately express the posterior probability distribution based on the measurement and control. In the standard particle filter algorithm, the prior probability density function is chosen as the proposed distribution. It can be calculated from the following formulation:

$$
q\left(x_{k}^{i} \mid x_{k-1}^{i}, z_{k}\right)=p\left(x_{k}^{i} \mid x_{k-1}^{i}\right)
$$

We simplify the weights and obtain:

$$
\omega_{k}^{i}=\omega_{k}^{i} / \Sigma_{t=1}^{N_{s}} \omega_{k}^{i}
$$

The posterior probability density can be expressed by 
$p\left(x_{k} \mid z_{1: k}\right) \approx \sum_{t=1}^{N_{s}} \omega_{k}^{i} \delta\left(x_{k}-x_{k}^{i}\right)$

When Ns is big enough, the calculation result is very close to the real posterior probability density.

\section{Research Directions of the Problem of SLAM}

Research on Real-time.In recent years, researchers have conducted a lot of research on autonomous mobile robot SLAM.On the one hand, to improve the existing SLAM algorithm, to improve the performance and feasibility of the algorithm, on the other hand the positive combination of robot sensors constantly updated equipment, proposed a new SLAM algorithm. But further research is needed in the following areas. By improving the efficiency of the algorithm and further reducing the time and space complexity of the algorithm, we can improve the real-time performance of the SLAM problem. At present, there are two types of map representation: geometric map and topological map. Although the geometric map is simple, the positioning accuracy is high, but its storage requirements are too high, and it is easy to produce the inconsistency of the map. Topological map can overcome these shortcomings, but the positioning accuracy cannot meet the actual requirements. In recent years, the research on the representation of attention mixed map. It has recently emerged in the multi-resolution and hierarchical map representation method is further extended to the topology map, this will be the future direction of the research on the map.

Research on Dynamics. The study of the outdoor natural environment is more difficult. At present, the problem of SLAM is limited to the research and application of two-dimensional static environment. It is of great significance to extend the research and application of SLAM from static environment to dynamic environment. The multi robot cooperation between robots and SLAM through information sharing based on complementary, can make up for a single robot positioning is not accurate and the composition of precision, but also improve the ability of autonomous mobile robot tasks in complex terrain. Compared with the indoor environment, outdoor environment is generally considered to be a dynamic environment, that is, the state of the environment will change over time. The existing static environment map building methods cannot be used in the dynamic environment, so it is more important to use the mobile robot to build a map in a dynamic environment. Because of the complexity and complexity of mobile robot map building, most of the research work is focused on the indoor dynamic environment. The key to realize intelligent autonomous mobile robot is to construct dynamic environment map online.

Research on Collaboration.Due to the unknown environment in the process of using multi robot than a single robot with high efficiency, and through the integration of different information obtained by robot, we can increase the modeling accuracy and robustness in recent years for cooperative localization, environment exploration and map building distributed multi robot system has become a hot research topic. These systems require the ability to share information across platforms, and integrate information from various sources into a consistent global map. Multi robot in addition to solve all the problems of single robot SLAM, but also to solve other problems caused multi robot system, including control structure, collaboration model, local sub map into the global map fusion algorithm. First of all, on the basis of the convergence of the single robot problem, the two important convergence properties of the multi robot cooperative problem are analyzed, which provides the theoretical basis for the existence of the problem. However, there are still a lot of problems in the multi robot cooperative SLAM, which can be attributed to the problem of distributed multi sensor information fusion, which is a direction to be studied in the future. In the multi robot system, each robot has its own coordinate system in the process, so the map created by different robots cannot be 
directly used for fusion. Scholars have developed a number of methods to achieve map fusion, usually, in the case of access to two types of prerequisites to achieve map fusion. The relative position of the robot is known to each other, such as the distance between the robot and the omnidirectional camera when the robot meets.

\section{Conclusions}

Simultaneous localization and mapping of mobile robots is a key problem in the field of robotics. In order to realize the autonomous navigation in the unknown environment, some breakthroughs of the three aspects of real-time, robustness and accuracy of the SLAM problem should be created in the following research. It is the key point to solve these problems to improve the efficiency of the algorithm and reduce the complexity of the algorithm.

\section{Acknowledgements}

This research is the result of the university level research project of Xi'an Peihua University named Development of Networking Remote Control Robot Based on the Embedded System(Grant No. PHKT16043).

\section{References}

[1] Luo Yuan, Yu Jiahang, Wang Longfeng, Wang Yunkai. Simultaneous localization and mapping of an improved RBPF based mobile robot [J]. CAAI Transactions on Intelligent Systems, 2015, 10(3):460-464.

[2] ZengPinshan, Miao Quanxing, Xu Lei. Research on Simultaneous Localization and Mapping Methods of Autonomous Mobile Robot [J]. Electronic Science and Technology, 2013, 26(9):177-178+181.

[3] Yan Deli, Song Yongduan, Song Yu, Kang Yifei. The application of square-root cubature Kalman filter andprobability hypothesis density in simultaneous localization andmapping for mobile robots [J]. Control Theory \& Applications, 2014, 31(8):1009-1017.

[4] Wang Fang, Liu Jin. Research of Simultaneous Location and Mapping of the Mobile Robot [J]. Intelligent Computer and Applications, 2015, 5(2): 73-75+79. 